\title{
Influence of urban morphology on air flow over building arrays
}

\author{
Matteo Carpentieri, Alan G. Robins \\ Environmental Flow Research Centre (EnFlo), University of Surrey, Guildford, UK, GU2 7HX
}

Accepted for publication in: Journal of Wind Engineering and Industrial Aerodynamics (2015)

\begin{abstract}
In the present paper we have analysed experimentally (wind tunnel) and numerically (CFD) the impact of some morphological parameters on the flow within and above the urban canopy. In particular, this study is a first attempt in systematically studying the flow in and above urban canopies using simplified, yet more realistic than a simple array of cuboids, building arrays. Current mathematical models would provide the same results for the six case studies presented here (two models by three wind directions), however the measured spatially averaged profiles are quite different from each other.

Results presented here highlight that the differences in the spatially averaged vertical profiles are actually significant in all six experimental/numerical cases. Besides the building height variability, other morphological features proved to be a significant factor in shaping flow and dispersion at the local to neighbourhood scale in the urban canopy and directly above: building aspect ratio (or, conversely, the street canyon aspect ratio), the angle between the street canyons and the incoming wind and local geometrical features such as, for example, the presence of much taller buildings immediately upwind of the studied area.
\end{abstract}

Keywords: Computational Fluid Dynamics; Flow field; Spatially averaged profiles; Turbulence profiles; Urban areas; Wind tunnel

\section{Introduction}

Air pollution in urban areas is an increasing concern, as the global urban population is growing in many countries. Recently, new concerns have arisen from the threat of accidental or deliberate release of hazardous gases in urban areas. There is a clear need of new mathematical tools capable of resolving the small spatial and temporal scales involved in the flow and dispersion phenomena in real complex cities. Computational fluid dynamics (CFD) models are currently capable of estimating the pollutant concentration field in smallto-medium sized spatial and temporal domains (Tominaga and Statopoulos, 2013), but they are very time-consuming and, for air quality management and emergency response purposes, much faster mathematical models are needed. Such fast, approximate models should be able to parameterise the relevant variables in a complex urban environment while, at the same time, providing acceptable results in terms of accuracy and reliability. 
High resolution urban models, especially at the local and intermediate (neighbourhood) scale, must take into account dispersion phenomena that occur in the urban canopy. The current approaches rely on empirical parametrisations derived from analytical studies and/or limited experimental data gathered mostly on very simplified geometries (e.g. single 2D street canyon or uniform arrays), or full scale measurements (usually very case specific). The influence of urban morphology on flow and dispersion in cities, and its parameterisation for urban flow and dispersion models has been studied, in the last couple of decades, either from a street canyon point of view (Theurer, 1999), or from a surface urban roughness point of view (Grimmond and Oke, 1999). The former relies on a description for the single street canyon based on canyon length $(L)$, width $(W)$ and height $(H)$. The latter relies on surface roughness $\left(z_{0}\right)$ and friction velocity $\left(u_{*}\right)$ estimated from parameters such as the mean building height $\left(H_{b}\right)$, the plan area index $\left(\lambda_{p}=A_{b} / A_{t}\right.$, where $A_{b}$ is the area occupied by the buildings, and $A_{t}$ is the total area) and the frontal area index $\left(\lambda_{f}=A_{f} / A_{t}\right.$, where $A_{f}$ is the frontal area of the buildings in a given vertical section, which obviously depends on location and wind direction). Recent developments in three-dimensional urban digital databases allow for automatic calculation of such parameters using, for example, image-processing techniques (Ratti et al., 2002; 2006).

Experimental and numerical studies have been carried out in order to characterise flow and dispersion in typical urban roughness configurations (see, e.g., MacDonald et al., 1998a; 2000). They have led to the development of the few urban canopy models that are available today. These models usually assume spatially averaged velocity profiles, adopting an approach similar to that used for flow over vegetation canopies (Finnigan, 2000; Coceal and Belcher, 2004), and, in some cases, even a single spatially averaged canopy velocity $\left(U_{C}\right.$, see Bentham and Britter, 2003). These properties depend strongly on the local geometry and existing models generally relate them to the mean building height $\left(H_{b}\right)$ and the lambda parameters $\left(\lambda_{p}\right.$, and $\lambda_{f}$, see above). Recent studies (Carpentieri et al. 2009, 2012a; Carpentieri and Robins, 2010; Harms et al., 2011; Klein et al., 2011), however, have highlighted the complexity of the flow and dispersion fields in actual urban geometries (as opposed to idealised building arrangements). It is clear from such studies that more parameters (such as building height variability and building aspect ratio) should be taken into account for a more accurate prediction of flow and dispersion in actual urban canopies.

Cheng and Castro (2002) performed wind tunnel experiments in building arrays with randomly distributed building heights, measuring velocity and turbulence in the roughness and inertial sub-layers. An interesting conclusion from their work is that cube arrays with variable height act as a substantially rougher surface than constant height arrays, even if the mean building height is the same. This is in contrast to all the current approaches described above where $z_{0}$ is only a function of mean building height and density. Xie and Castro (2006) and Xie et al. (2008) studied the same configurations by simulating the flow with both Reynolds-averaged Navier-Stokes (RANS) and large-eddy simulation (LES) CFD models. Their 
results showed that many features of the flow over the variable-height array are rather different from those in the flow over uniform roughness. They concluded that generalising modelling approaches derived from simpler (uniform) arrays is not a viable option for urbanlike arrays, and more experimental and computational studies on this aspect are needed.

Another important aspect of the local urban geometry, the building aspect ratio (length : width : height ), has received less attention. Most of the systematic studies on building arrays involve the use of cuboidal obstacles (Cheng and Castro, 2002; Cheng et al., 2007) or relatively deep and long street canyons (Kastner-Klein and Plate, 1999; Salizzoni et al., 2009). The few papers that consider a more realistic urban form usually tend to focus on diagonally symmetric buildings (width = length), so that fewer wind directions are needed to completely characterise flow and dispersion (Garbero et al., 2010). Building aspect ratios are expected to play a significant role in so-called 'topological dispersion' (Davidson et al. 1995, Jerram et al. 1995, Belcher 2005), where the presence of the obstacle (building) enhances the lateral dispersion of the plume. This effect is greatly enhanced for some wind directions when the building has a form other than the classic cuboid, as evidenced in the few studies involving such types of buildings (see, e.g., MacDonald, 1998b; Yee and Biltoft, 2004; Milliez and Carissimo, 2007).

The present study has been carried out in the framework of the HRModUrb project (High Resolution Models for Flow and Pollutant Dispersion in Urban Areas), funded by the European Commission under the FP7 - People Programme (Marie-Curie Actions). The overall objective was to study the effects of urban morphology on flow and dispersion phenomena at the local and neighbourhood scales, addressing the above issues. In the present paper, the effects of these parameters on the spatially averaged velocity profiles will be investigated through a series of systematic wind tunnel experiments, partially supported by some numerical CFD simulations

The specific objectives of the present study include:

1. Measuring vertical wind profiles in urban models with different morphological characteristics;

2. Assessing the influence of building height variability;

3. Investigating the influence of building aspect ratio with respect to wind direction;

4. Evaluating the representativeness of spatially averaged wind profiles and canopy averaged velocities. 


\section{Wind tunnel experiments}

\subsection{The models}

The aim of the present study was to investigate the effect of morphology on flow and dispersion in realistic urban environments. In order to do this, the development of urban models more complex than the usual array of cubic buildings was necessary. However, the models had to be simple enough that a systematic study and a relatively easy parameterisation could be possible.

The starting point for the design of the models was the 1:200 scale model of the DAPPLE field site in central London (Arnold et al. 2004, Carpentieri et al. 2009). A substantial amount of data has been gathered in wind tunnel experiments, field tests and numerical simulations for the DAPPLE site, and these data can be used in future as comparison for the simpler models.

The models designed for this study are again at a nominal scale of 1:200 and have two main intersecting streets (approximately matching those of the DAPPLE site: Marylebone Road, along the $x$ axis, and Gloucester Place, along the $y$ axis) and several smaller streets. The dimensions (width) of the main streets are, respectively: $220 \mathrm{~mm}$ and $110 \mathrm{~mm}$ (44 and $22 \mathrm{~m}$ at full scale). The building blocks occupy an area of $230 \times 350 \mathrm{~mm}^{2}$ (arranged with the longer dimension along the $y$ axis). In order to match the DAPPLE site $\lambda_{p}=0.54$, an array of $6 \times 8$ buildings was built, with the width of the secondary streets equal to $99 \mathrm{~mm}$ (see figure 1). 


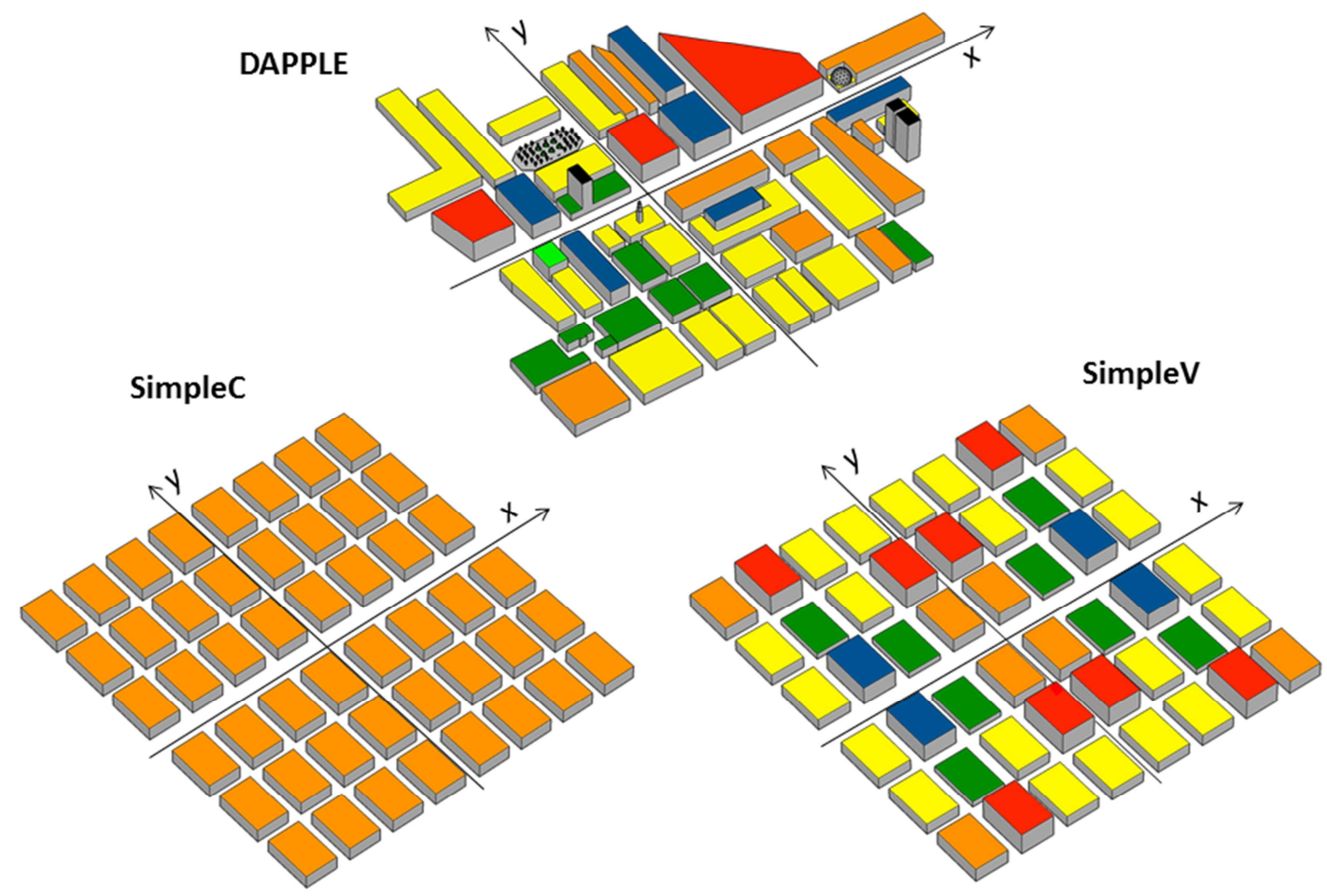

Figure $13 D$ representation of the models: DAPPLE (top), SimpleC (left) and SimpleV (right); the colours represent the building height range: green $(55-75 \mathrm{~mm})$, yellow $(75-95 \mathrm{~mm})$, orange (95-115 mm), blue (115-155 $\mathrm{mm})$ and red (155-170 $\mathrm{mm})$.

Two models were employed in order to investigate the influence of the building height variability on the flow and dispersion phenomena, one with constant building height and the other variable building height. The simplest model (named 'SimpleC') had a constant building height $\left(H_{b}=102 \mathrm{~mm}\right.$, which is the mean building height of the central part of the DAPPLE model). The other model ('SimpleV') was designed with five different building heights. The height of the DAPPLE model buildings were divided into five classes of height ranges (55-75 mm, 75-95 mm, 95-115 mm, 115-155 mm, 155-170 mm) and the distribution of the building heights in SimpleV matched that of the DAPPLE model. A building height of $102 \mathrm{~mm}$ was chosen for the $95-115 \mathrm{~mm}$ class for practical convenience. The other building heights were adjusted in order to give the required overall mean height (i.e. $102 \mathrm{~mm}$ ), $\lambda_{f}$ ( 0.24 for wind direction parallel to the $x$ axis, and 0.16 for wind direction parallel to the $y$ axis) and height variability $\left(\sigma_{H}=32 \mathrm{~mm}\right)$ as the DAPPLE model. It comprises the following buildings: $8 \times H_{1}(65 \mathrm{~mm}), 20 \times H_{2}(85 \mathrm{~mm}), 8 \times H_{3}(102 \mathrm{~mm}), 4 \times H_{4}(135 \mathrm{~mm})$, and $8 \times H_{5}$ $(162 \mathrm{~mm})$. The distribution of the different buildings in the model was defined to provide a symmetric distribution along both axes, with the four central buildings chosen among the $\mathrm{H}_{3}$ class, in order to have similarity in the central part of the model between SimpleC and SimpleV. 
The coordinate system used throughout the paper is aligned with the models. The $x$ axis is always parallel to the largest street, with the $y$ axis perpendicular to it with an origin in the centre of the model (see also Figures 2 and 3 ).

\subsection{Experimental strategy}

The experiments were carried out at the boundary layer wind tunnel of the Environmental Flow Research Centre (EnFlo), University of Surrey, UK. It is an open circuit 'suck-down' wind tunnel with a $20 \mathrm{~m}$ long, $3.5 \mathrm{~m}$ wide and $1.5 \mathrm{~m}$ high working section. The wind speed can be in the range 0.3 to $3.5 \mathrm{~m} \mathrm{~s}^{-1}$, and the facility is capable of simulating both stable and unstable atmospheric conditions, although this feature was not used in this study. Reference flow conditions are measured by two ultrasonic anemometers, one held at a fixed location and the other positioned as required, and two propeller anemometers mounted on either side of the traverse carriage; the motor shaft speed is also measured. Temperature conditions are monitored by thermocouple rakes in the flow and individual thermocouples in each tunnel wall panel. The pressure drop across the inlet is also monitored, primarily to indicate the state of the inlet screens. The wind tunnel and the associated instrumentation are fully automated and controlled using 'virtual instrument' software created by EnFlo research staff using LabVIEW.

The resulting blockage ratios for the SimpleC models are respectively $2.5 \%, 1.7 \%$ and less than $6.6 \%$, depending on the wind direction. Of course the 45 degree wind direction value is only an upper limit, since the calculation of the actual blockage ration is not trivial in this case. This is also the case for the SimpleV model, where the upper limits for the blockage ratios are, respectively, $3.8 \%, 2.1 \%$ and $9.6 \%$ (note that the $45^{\circ}$ value should be significantly lower than the upper limit).

Flow measurements were performed by means of laser Doppler anemometry (LDA). The EnFlo laboratory is equipped with a dual beam, optical heterodyne detection system, manufactured by DANTEC. An ultrasonic haze generator is used to produce particles suitable for the LDA measurements. With this method, ultrasonic transducers in a shallow tank of fluid create $\sim 2 \mu \mathrm{m}$ size droplets which are blown away from the surface by a fan. The type of fluid used (a mix of water and sugar) has a high refractive index, to ensure that the seeding has good light scattering properties.

Before the actual execution of the experimental plan, some preliminary measurements were made in order to validate the various measurement methodologies and test the wind tunnel set-up. In addition, a set of measurements was deemed necessary to feed the numerical simulations (see section 3 ) with high quality data to be applied as boundary 
conditions. These preliminary tests were all performed on the SimpleC model mounted in the wind tunnel in the $0^{\circ}$ configuration (wind direction along the $x$ axis). For the boundary conditions tests, the measurements were also repeated with an empty tunnel to assess the influence of the model on the approach flow, though the results only showed variations within the measurement uncertainty range expected in this kind of experiments. All the preliminary tests were run using a reference wind speed measured above the boundary layer ( $U_{\text {ref, }}$ measured at $1 \mathrm{~m}$ height) of $2.5 \mathrm{~m} \mathrm{~s}^{-1}$ (the 'free stream velocity'). The Reynolds number based on the mean building height in the experimental conditions was $R e \approx 1.7 \cdot 10^{4}$.

Vertical mean flow and turbulence profiles (which could be used to derive spatially averaged vertical profiles and average canopy velocity) were measured by means of the twocomponent LDA ( $x$ and $y$ components of the mean velocity, respectively $U$ and $V$, and of the velocity fluctuations, $u$ and $v$ ). Two different spatial arrangements of the measurement grid were tested: the first grid was focussed on the central intersection, while the second grid covered a wider area with a lower spatial resolution. Both the SimpleC and SimpleV models were tested. The measurements were carried out with a reference wind speed of $2.5 \mathrm{~m} \mathrm{~s}^{-1}$ for three wind directions (model rotations: $0^{\circ}, 90^{\circ}$ and $45^{\circ}$ ). The same averaging time of about 1 minute as in previous experiments within the DAPPLE model (Carpentieri et al. 2009) was used. The measurement grid for the smaller (high resolution) domain is shown in figure 2. Vertical profiles were measured up to approximately $3 H_{b}$. The measurement grid for the wider (lower resolution) domain is shown in figure 3. Vertical profiles, in this latter case, were measured up to approximately $6 H_{b}$. 

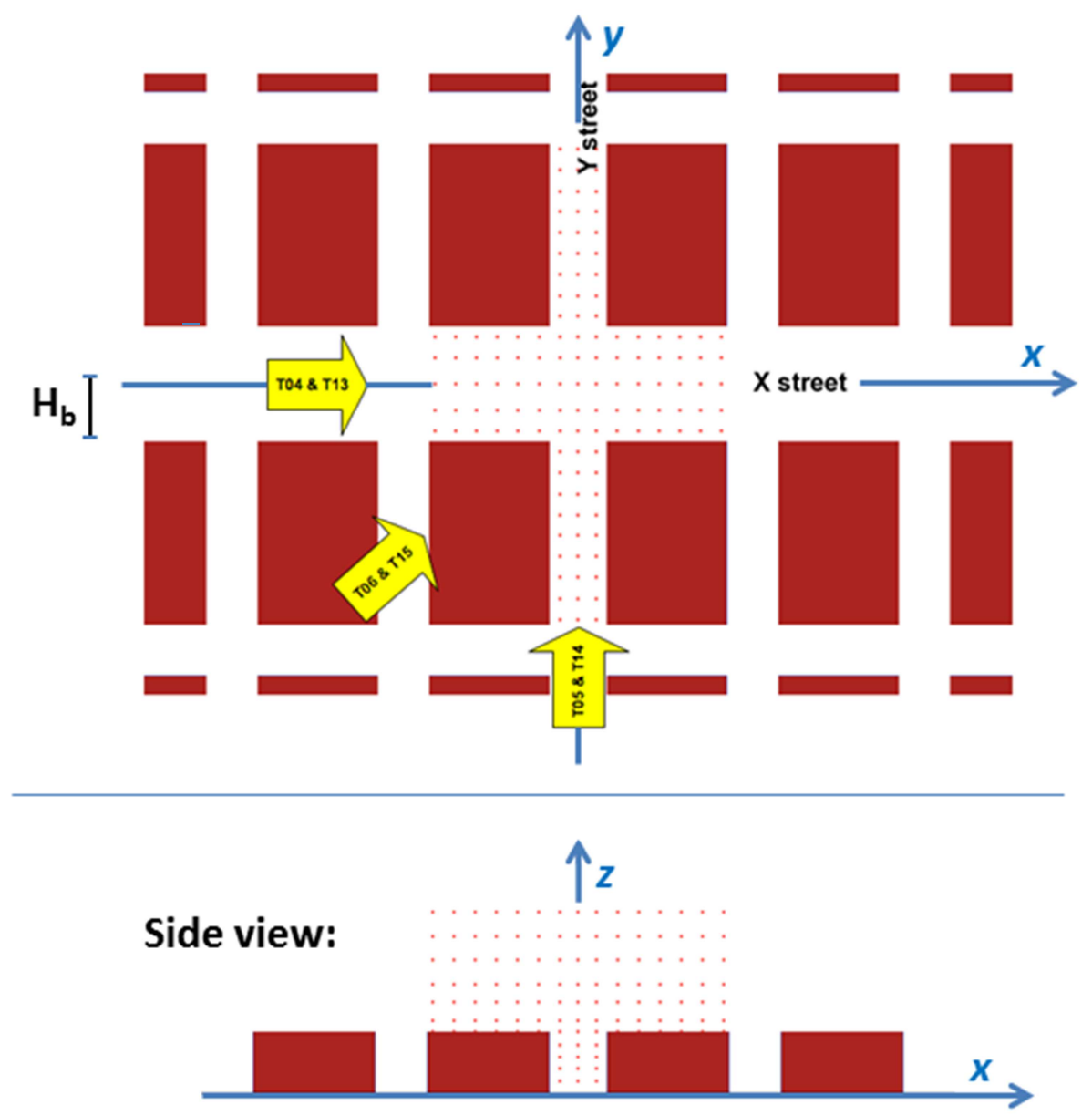

Figure 2 LDA measurement grid (smaller domain); $x-y$ plane (top), and $x$-z plane (bottom); the length scale $H_{b}$ (mean building height $=102 \mathrm{~mm}$ ) is also reported on the map; only the central area of the model is shown here. 


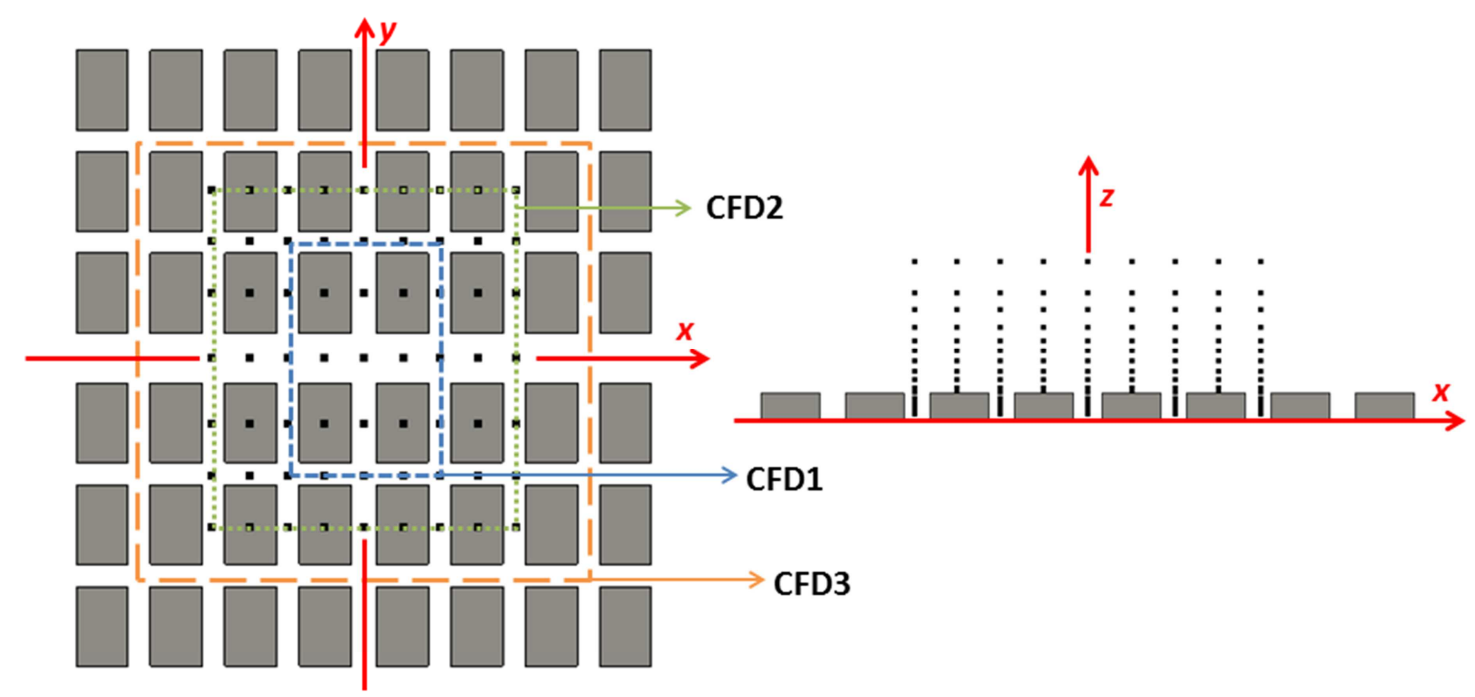

Figure 3 Black dots represent the LDA measurement grid (wider domain); $x-y$ plane (left), and $x-z$ plane (right); the extent of the various CFD space averaging domains are also shown; the side view refers to the SimpleC model: the grid used for SimpleV is similar, accounting for the different building heights.

A summary of the wind tunnel tests performed in the present study is reported in table 1. 
Table 1 Summary of wind tunnel tests; 'LDA-1' refers to the high resolution smaller grid, 'LDA-2' to the low resolution large grid, 'Prel' to the vertical profiles for the preliminary measurements, and 'CFD-BCs' to the measured points for the CFD boundary conditions

\begin{tabular}{llllll}
\hline Test & Instrument & Measured & Model & WindDir & Grid \\
\hline T01 & LDA & $U, V$ & SimpleC & $0^{\circ}$ & Prel \\
T04 & LDA & $U, V$ & SimpleC & $0^{\circ}$ & LDA-1 \\
T05 & LDA & $U, V$ & SimpleC & $90^{\circ}$ & LDA-1 \\
T06 & LDA & $U, V$ & SimpleC & $45^{\circ}$ & LDA-1 \\
T13 & LDA & $U, V$ & SimpleV & $0^{\circ}$ & LDA-1 \\
T14 & LDA & $U, V$ & SimpleV & $90^{\circ}$ & LDA-1 \\
T15 & LDA & $U, V$ & SimpleV & $45^{\circ}$ & LDA-1 \\
T31 & LDA & $U, V, W$ & SimpleC + & $0^{\circ}$ & CFD-BCs \\
& & & empty tunnel & & \\
T32 & LDA & $U, V$ & SimpleC & $0^{\circ}$ & LDA-2 \\
T33 & LDA & $U, V$ & SimpleC & $90^{\circ}$ & LDA-2 \\
T34 & LDA & $U, V$ & SimpleC & $45^{\circ}$ & LDA-2 \\
T38 & LDA & $U, V$ & SimpleV & $0^{\circ}$ & LDA-2 \\
T39 & LDA & $U, V$ & SimpleV & $90^{\circ}$ & LDA-2 \\
T40 & LDA & $U, V$ & SimpleV & $45^{\circ}$ & LDA-2 \\
\hline
\end{tabular}

\section{CFD simulations}

CFD simulations were also carried out in order to integrate and, eventually, extend the wind tunnel experimental data. This hybrid approach, often referred to as the 'C-FD-E approach' (Hangan, 1999; Robins, 2003), combines the advantages of the two techniques, establishing a reciprocal feedback.

The geometry of the models for the numerical simulations was as similar as possible to the wind tunnel experiments (see section 2.1). In order to meet the requirements for the standard wall functions on the non-dimensional distance $\left(z^{+} \geq 30\right)$, the models were scaled up from 1:200 (wind tunnel size) to 1:50. This technique has already been used in the past in similar applications (see for example Hamlyn and Britter, 2005). The resulting Reynolds number based on the mean building height for the CFD simulations was $R e \approx 6.8 \cdot 10^{4}$.

The domain size for the various test cases was chosen following the few existing guidelines on atmospheric flows in urban areas (Franke et al., 2007; Tominaga et al., 2008). In particular, in the $0^{\circ}$ and $90^{\circ}$ cases, the upwind fetch from the inlet boundary to the first 
obstacle row was set to $5 H_{b}$, while the downwind distance from the last building to the outlet boundary was set conservatively to $15 H_{b}$. The lateral boundaries in these cases were located at the same location as the wind tunnel walls. The $45^{\circ}$ case was modelled using the same coordinate system for the computational mesh, so that we had two inlet boundaries (located at $\sim 5 H_{b}$ from the buildings), two outlet boundaries (at $\sim 10 H_{b}$ ) and no lateral boundaries. In all cases the top boundary was located at $z=6.86 \mathrm{H}_{b}$. The resulting horizontal domain sizes were $44.85 H_{\mathrm{b}} \times 34.31 H_{\mathrm{b}}, 34.31 H_{\mathrm{b}} \times 46.57 H_{\mathrm{b}}$ and $39.95 H_{\mathrm{b}} \times 41.67 H_{\mathrm{b}}$, respectively for the $0^{\circ}, 90^{\circ}$, and $45^{\circ}$ wind directions. The blockage ratios in the CFD model were slightly larger for the $0^{\circ}$ and $90^{\circ}$ wind directions (due to the smaller height, compared to the wind tunnel: $3.35 \%$ and $2.28 \%$ (SimpleC), and less than $5.09 \%$ and $2.81 \%$ (SimpleV). The blockage ratios for the $45^{\circ}$ cases were, on the contrary, smaller than for the wind tunnel models due to the fact that the domain section was larger.

A high resolution graded Cartesian mesh with $\sim 1.8$ to $\sim 2.3$ million cells, depending on the particular case considered, was generated, with increasing spatial resolution close to the buildings and the ground. The number of surface cells across the $Y$ street canyon was 11 , with 8 cells across the smaller canyons and 22 across $X$ street. The number of cells over the height of the intermediate building (the central one) edge was 10 . A representation of the domain and grid for the SimpleC case at $0^{\circ}$ is reported in Figure 4 as an example.

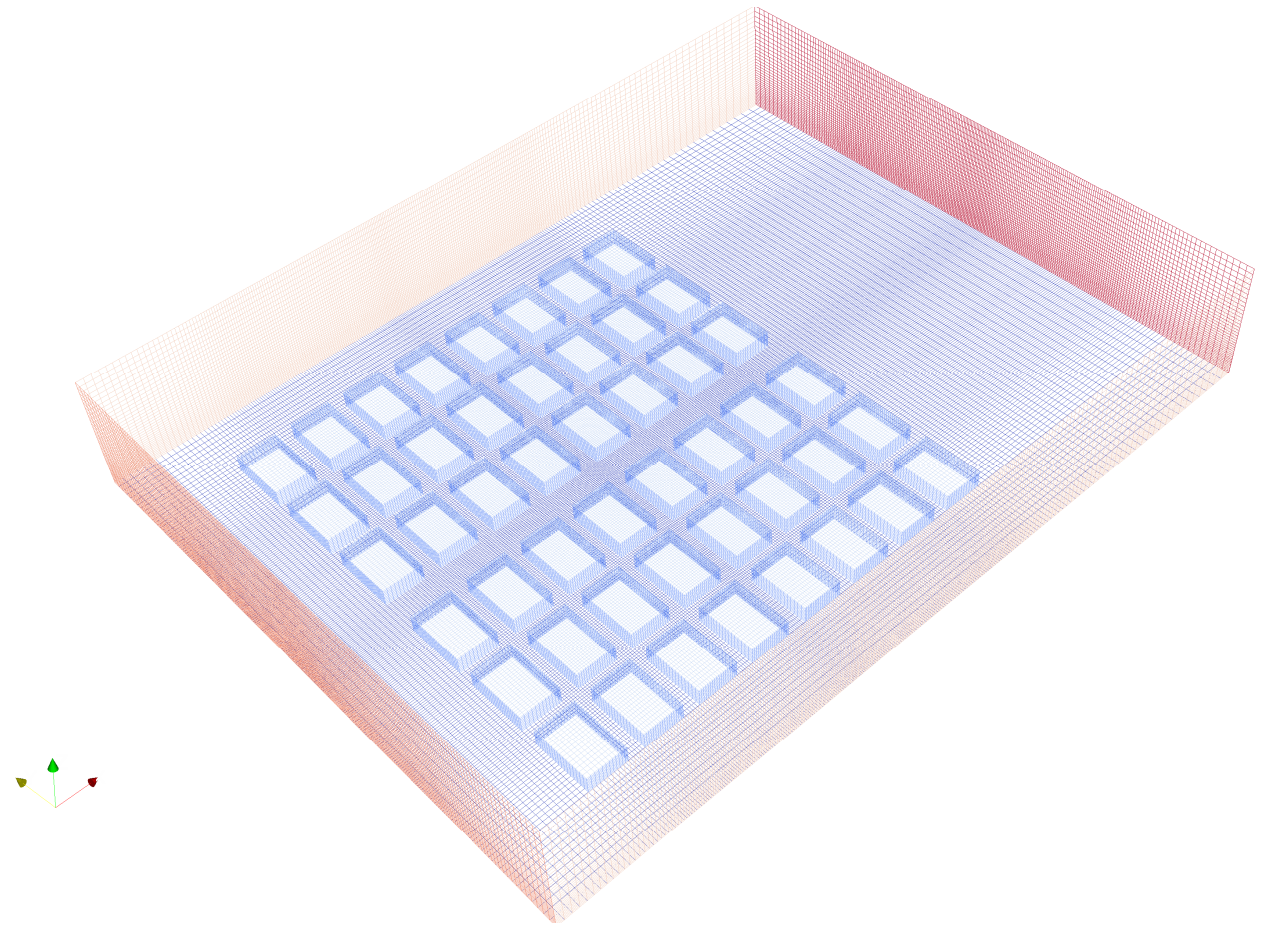

Figure 4 Rendering of the CFD domain and grid for the SimpleC model at $0^{\circ}$ wind direction. Similar grids were used for the other test cases.

All the solid surfaces were treated using standard wall functions (see, e.g., Blocken et al., 2007). The same wall functions were applied to the lateral boundaries in the 0 and $90^{\circ}$ cases. The top boundary was treated as a symmetry plane. An open boundary condition was 
used at the outlet. The inlet boundary condition was characterised by using the wind tunnel measurements mentioned in section 2.2. Some of the measured profiles are shown in figure 5 . The turbulent dissipation rate profile $(\varepsilon)$ was estimated:

$$
\varepsilon=\frac{u_{*}^{3}}{\kappa z}
$$

where $u_{*}$ is the friction velocity derived from the logarithmic fit to the mean velocity measurements in figure $5, k$ is the Von Karman constant, and $z$ is the height above the ground. The estimated values for the roughness length and friction velocity at the inlet profile were, respectively, $z_{0}=0.015 H_{\mathrm{b}}$ and $\mathrm{u}_{*}=0.06 U_{\text {ref. }}$.
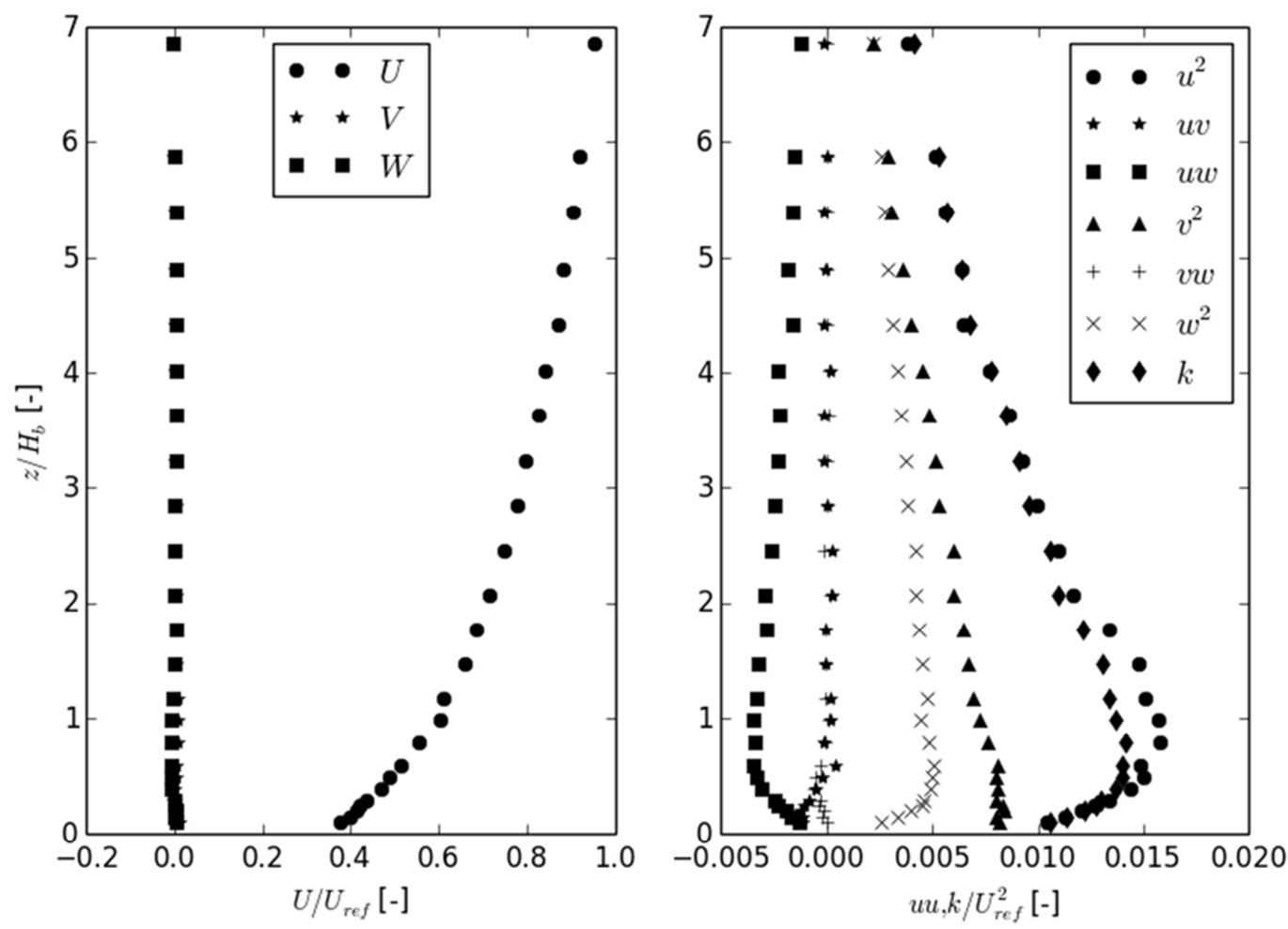

Figure 5 Measured inlet velocity (left) and turbulence (right) profiles; all the values are nondimensionalised using the free stream velocity $U_{r e f} ; k$ is the turbulent kinetic energy, calculated from the three components of turbulence: $k=1 / 2\left(u^{2}+v^{2}+w^{2}\right)$.

All the numerical simulations were carried out using the OpenFOAM C++ open source library (OpenFOAM, 2009). The RANS approach, with the standard $k-\varepsilon$ turbulence model (Launder and Spalding, 1974), was used in this particular study. Steady simulations were carried out, giving a time-averaged view of the flow, neglecting possible phenomena such as unsteady vortex shedding and intermittent sweeps of air from above the canopy (Hamlyn and Britter, 2005). The equations were solved by using the SIMPLE algorithm (Semi-Implicit Method for Pressure-Linked Equations, Caretto et al., 1973). A second-order upwind difference scheme 
in space was applied, while the linearised system was solved using the Multi-Grid method. The numerical calculations were considered converged when the normalised residuals were below $10^{-5}$ for pressure and $10^{-6}$ for all other variables.

A preliminary comparison between CFD results and wind tunnel measurements was carried out for the T04 test case (see table 1), with the SimpleC model and $0^{\circ}$ wind direction. The comparison showed excellent agreement between measured and simulated data for the variables of interest (velocity and turbulence). A statistics summary (using the indices: fractional bias, FB, normalised mean standard error, NMSE, correlation coefficient, $R$ and ratios within a factor of 2, FAC2, as described by Chang and Hanna, 2004) is reported in Table 2. The indices were calculated as described below.

FB (fractional bias) is defined as:

$$
F B=2 \frac{\overline{\chi^{0}}-\overline{\chi^{s}}}{\overline{\chi^{o}}+\overline{\chi^{s}}}
$$

where $\chi^{0}$ is the measured value of the variable of interest, $\chi^{5}$ is the calculated value of the variable of interest, and the overbar indicates the average over all the available points; it ranges between -2 and +2 , a perfect model would give $F B=0$, while if $F B>0(<0)$ the model on average underestimates (overestimates) the observed concentrations.

NMSE (normalised mean square error) is defined as:

$$
N M S E=\frac{\overline{\left(\chi^{o}-\chi^{s}\right)^{2}}}{\overline{\chi^{0}} \overline{\chi^{s}}}
$$

a perfect model would give NMSE $=0$, the value of this index is always positive.

$\mathrm{R}$ (linear correlation coefficient) is defined as:

$$
R=\frac{\overline{\left(\chi^{o}-\overline{\chi^{o}}\right)\left(\chi^{s}-\overline{\chi^{s}}\right)}}{\sigma^{o} \sigma^{s}}
$$

where $\sigma^{\circ}$ and $\sigma^{\mathrm{s}}$ are the standard deviations of, respectively, the observed and simulated values of the variable of interest; a perfect model would give $R=+1$, it ranges between -1 and +1 .

FAC2 (fraction within a factor of 2 ) is defined as:

$$
\text { fraction of data with } 0.5 \leq \frac{\chi^{s}}{\chi^{o}} \leq 2
$$

a perfect model would give $\mathrm{FAC2}=1$. 
Table 2 Summary of comparison statistics between CFD results and wind tunnel measurements for the T04 case; $U$ is the $x$ component of the velocity vector, while $k$ is the turbulent kinetic energy

\begin{tabular}{lllll}
\hline Parameter & FB & NMSE & R & FAC2 \\
\hline $\boldsymbol{U}$ & -0.05 & 0.01 & 0.99 & 0.93 \\
$\boldsymbol{k}$ & 0.23 & 0.11 & 0.73 & 0.90 \\
\hline
\end{tabular}

\section{Results}

Examples from the full data-base for the cases listed in Table 1 are discussed below, chosen to demonstrate features of the overall data-set. The full data-set is available from the authors.

\subsection{Building height variability}

Selected vertical profiles of the horizontal velocity, $V$, and turbulence component, $v^{2}$, in the SimpleC and the SimpleV models are presented in figures 6-8.
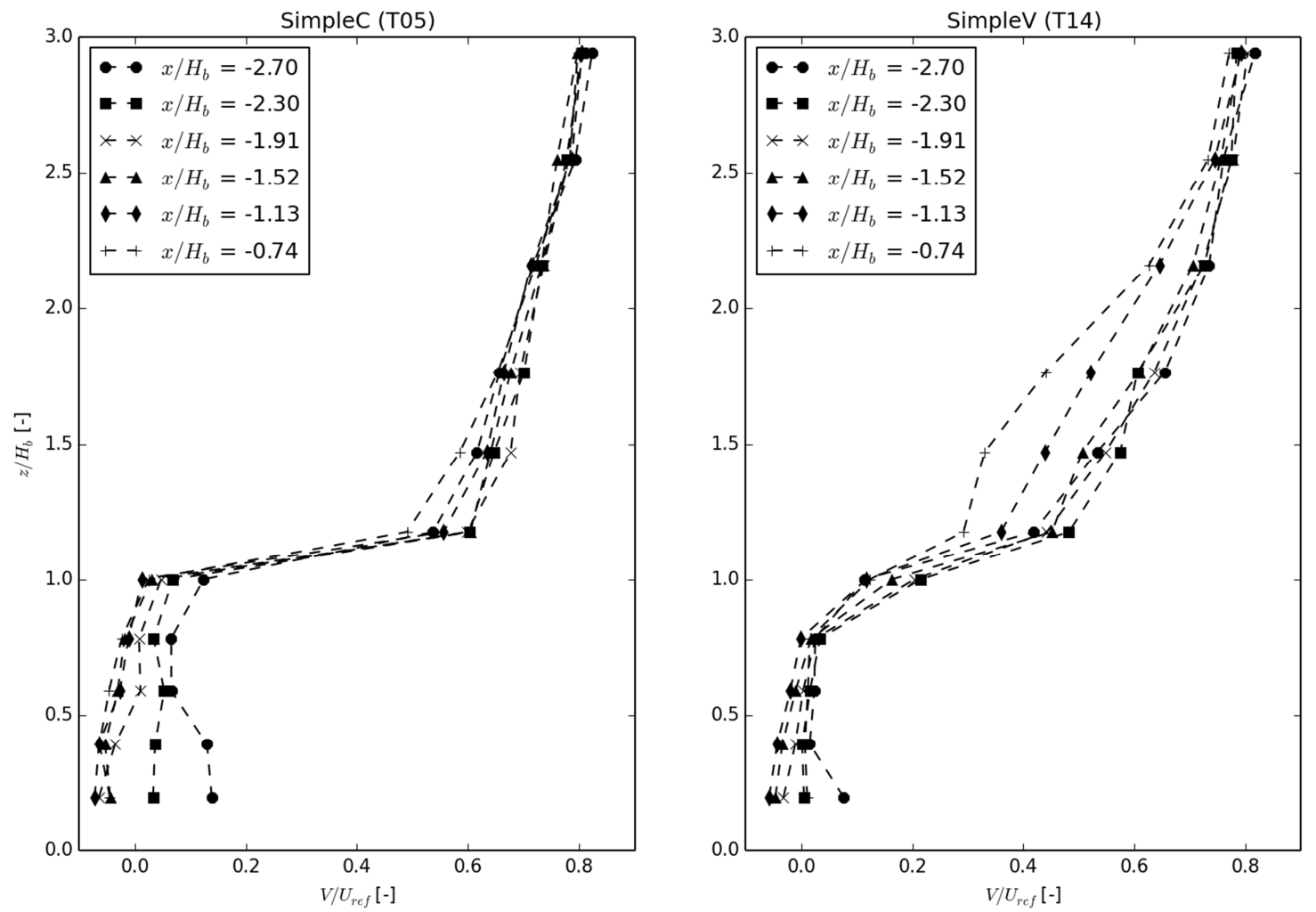

Figure 6 Vertical profiles of the mean horizontal velocity at $y / H_{b}=-0.88$ (profiles closest to the upwind building), wind direction $90^{\circ}$, for SimpleC (T05; left) and SimpleV (T14; right), wind tunnel measurements; velocities are non-dimensionalised by the free stream velocity $U_{\text {ref; }}$ the maximum building height in SimpleV is at $1.59 H_{\mathrm{b}}$ 

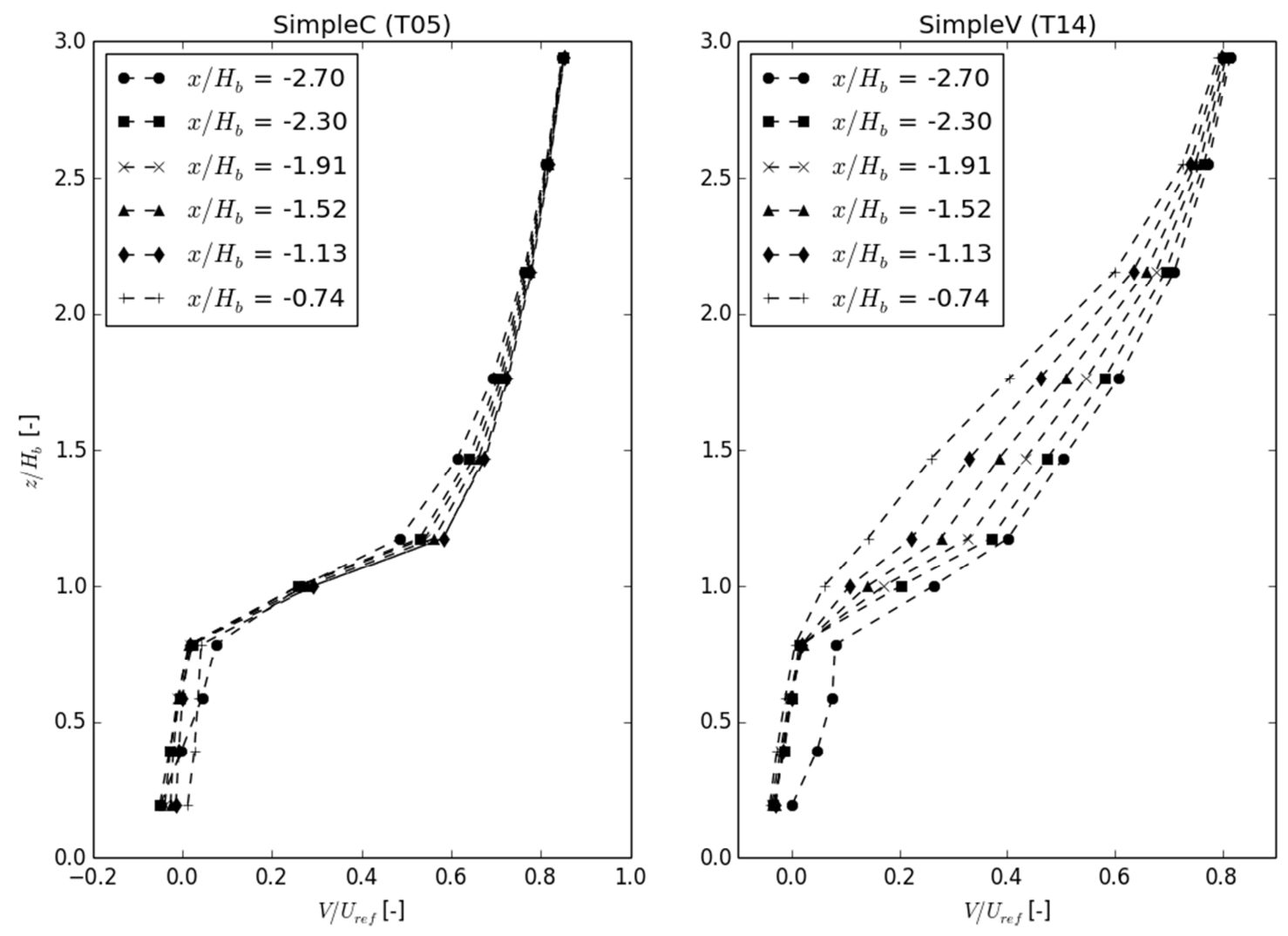

Figure 7 Vertical profiles of the mean horizontal velocity at $y / H_{b}=-0.88$ (profiles closest to the upwind building), wind direction $90^{\circ}$, for SimpleC (T05; left) and SimpleV (T14; right),CFD simulations; velocities are non-dimensionalised by the free stream velocity; the maximum building height in SimpleV is at $1.59 \mathrm{H}_{\mathrm{b}}$ 

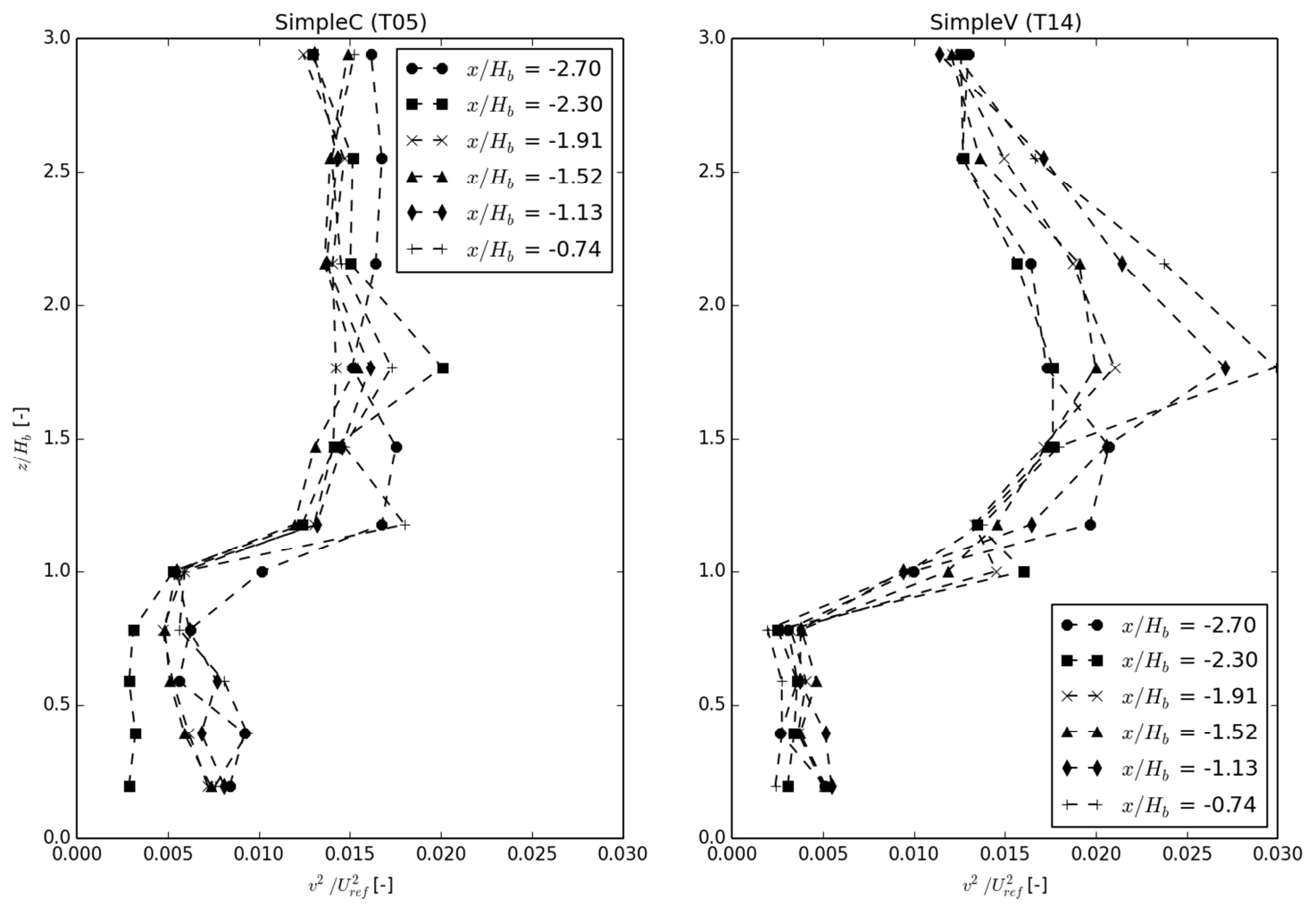

Figure 8 Vertical profiles of the along-wind component of turbulence at $y / H_{b}=-0.88$ (profiles closest to the upwind building), wind direction $90^{\circ}$, for SimpleC (T05; left) and SimpleV (T14; right), wind tunnel measurements; velocities are non-dimensionalised by the free stream velocity $U_{\text {ref; }}$ the maximum building height in SimpleV is at $1.59 \mathrm{H}_{\mathrm{b}}$

Generally, results from the SimpleV model at wind directions of $0^{\circ}$ and $90^{\circ}$ ( $\mathrm{T} 13$ and $\mathrm{T} 14$ ) are quite similar to those from SimpleC (T04 and T05). The building height variability induces more variablity in the mean velocity profiles, especially in street canyons that are perpendicular to the approach-flow (i.e. the Y Street in Case T13 and the X Street in T14; the latter is shown in figures 6 and 7), also because the shear layer is larger in SimpleV. However, a clear difference is visible in the turbulence peaks (Figure 8); along-wind turbulence values ( $u$ for T04 and T13, $v$ for T05 and T14) show different levels below and above roof level, with in-canopy turbulence much less than above-canopy. A turbulence peak approximately at roof level can be observed in both models (the buildings around the main intersection are $102 \mathrm{~mm}$ high also in the SimpleV model), but in the SimpleV case the turbulence profiles are more complex, with multiple, usually weaker, turbulence peaks due to the influence of upstream buildings of variable height. This is particularly evident for Case T14 (Figure 8), where the buildings upstream of the 'intersection blocks' are significantly taller than the four buildings around the main intersection (see Figure 1 - right). No general comments can be made for the $45^{\circ}$ wind direction cases (T06 and T15), although the complexities in the turbulence profiles are clearly enhanced in the Case T15 results, due to 
the influence of a number of upstream buildings with different height. Further discussion on the influence of wind direction can be found in Section 4.2.

The increased levels of turbulence just above the urban canopy will obviously affect dispersion process in at least in two ways: (1) the exchange of pollutants between the street canyon and the flow above will likely increase; (2) an enhanced turbulence field above the urban canopy will enhance dispersion above roof level, affecting the pollutant concentration field at the neighbourhood scale. As already stated in Section 1, there is no current model capable of taking into account the influence of the building height variability on the dispersion process, leading to an underestimation of pollutant dispersion in urban areas with a more heterogeneous texture.

Spatially averaged profiles were calculated using the datasets discussed above:

$$
U\left(z_{i}\right)=\frac{\sum_{j=1}^{N_{i}} U_{j}\left(z_{i}\right)}{N_{i}}
$$

where $\mathrm{U}$ is the averaged along-wind mean velocity component, $z_{\mathrm{i}}$ are the heights at which the wind speed has been measured (or calculated, in the case of CFD simulations), $N_{i}$ the number of measurements (or calculated values) at a particular height and $U_{j}$ the individual measurements (or numerical estimates) of along-wind mean velocity component.

The results from the wind tunnel measurements are shown in Figure 9, for the smaller highresolution domain. The standard deviations in Figures 9 (right), 10 (right) and 11 (right) were calculated as:

$$
\sigma_{U}\left(z_{i}\right)=\sqrt{\frac{\sum_{j=1}^{N_{i}}\left[U_{j}\left(z_{i}\right)-U\left(z_{i}\right)\right]^{2}}{N_{i}-1}}
$$



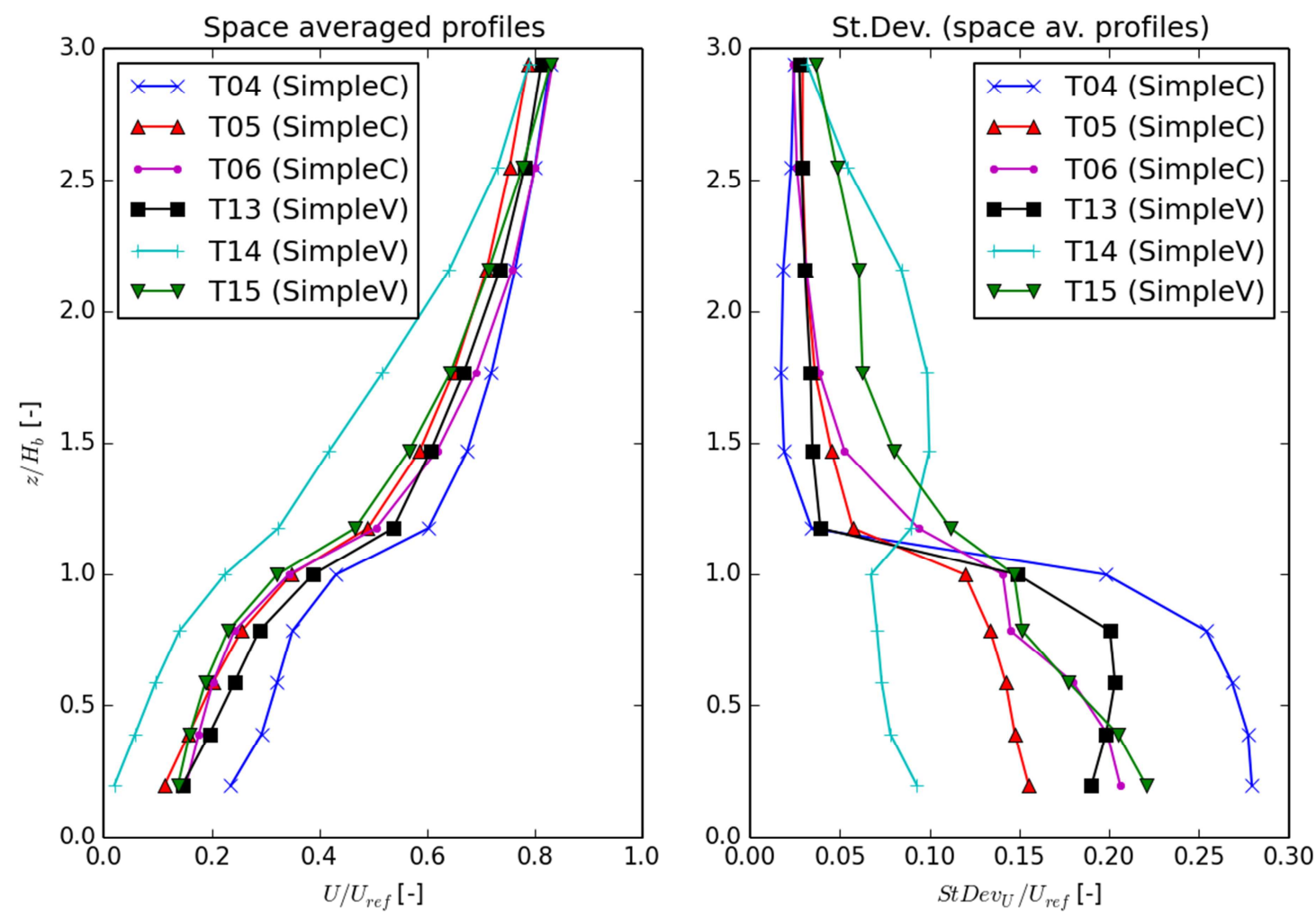

Figure 9 Spatially averaged vertical profiles of velocity (left), and standard deviation of the average profile (right); wind tunnel measurements in the smaller high-resolution domain; the maximum building height in SimpleV is at $1.59 \mathrm{H}_{\mathrm{b}}$

The differences in the geometry and the arrangement of the buildings are reflected in the differences in the averaged profiles and shown in Figure 9-left. Marked differences can be found both in the in-canopy and above-canopy parts of the profiles. Figure 9-right shows the spatial standard deviations of the averaged profiles. As expected, a very high variability is found within the canopy, while the velocity profiles tend to be more uniform in the roughness sub-layer, above roof level.

The differences in the velocity profiles decrease with height, as expected. At $z=300 \mathrm{~mm}$ $\left(z / H_{b}=2.94\right)$ these differences are quite small, since the influence of the model geometry is weak at that height. This fact is also reflected in the shape of the standard deviation profiles in Figure 9-right. The shape of the velocity profiles is similar in all cases, as is also true of the standard deviation profiles, even though the numerical values are different in the various tests. The only exception is Case T14 (model SimpleV with $90^{\circ}$ wind direction) where the presence of two very tall buildings (height $=162 \mathrm{~mm}$ ) just upwind of the measurement domain enhances the variability of the vertical profiles even well above the street canopy, reinforcing the conclusion that local geometrical features can sometimes have a larger impact on urban wind profiles than larger scale geometrical characteristics. 
The spatially averaged profiles for the $0^{\circ}$ (Cases T04 and T13) and 90 (Cases T05 and T14) wind directions show a generally higher flow speed over the SimpleC model, both above and within the canopy. The standard deviation of the spatially averaged profiles, which can be thought as a measure of how much a single vertical profile may differ from the averaged, is also much larger for the T04 and T05 cases when compared with T13 and T14, particularly within the street canopy. Above the roofs the situation is completely different, with an increased variability in the vertical profiles shown by the SimpleV cases. On the contrary, the difference in spatially averaged vertical profiles between SimpleC and SimpleV tends to be much smaller in the $45^{\circ}$ wind direction cases (T06 and T15); the standard deviations are also similar as in this situation, the influence of the building height variability on the flow field is reduced.

\subsection{Building aspect ratio and wind direction}

Analysis of the influence of building aspect ratio and wind direction on the flow within and above the urban canopy starts by comparing the results for the constant height case (SimpleC, T04, T05 and T06). Figure 9 (left) shows little difference between the $90^{\circ}$ case and the $45^{\circ}$ case, while the wind speeds for the $0^{\circ}$ case seem to be generally higher, especially within the canopy. The main reason for this result is probably that in this case the wind direction is aligned with the wider $X$ Street, where greater velocities are expected.

The wind profiles seem to be, again, more correlated with local geometrical features than with the frontal area density, as one might expect. In fact the highest $\lambda_{f}$ value is found for T06 (0.28), while in T04 and T05 the values are, respectively, 0.24 and 0.16 . From this point of view, it might be surprising (without taking into account the impact of local geometrical features) that the most similar profiles are found for cases with the largest difference in frontal area density $(0.16$ versus 0.28$)$.

The above pattern generally holds for the SimpleV experiments as well. In this case, though, the differences between the $0^{\circ}$ case and the $45^{\circ}$ case are less pronounced, while the average wind profile for the $90^{\circ}$ case (T14) shows much reduced values. In this case the dominant feature becomes the presence of the two very tall buildings upwind of the intersection, as explained in the previous section.

The wind tunnel data are generally confirmed by the CFD simulations shown in Figure 10, even if the differences between the spatially averaged profiles appear to be larger than in the wind tunnel data. The spatial averages have been calculated using the same locations as in the wind profiles in the smaller high-resolution domain in the wind tunnel experiments. The influence of the spatial domain and the number of measurement points on the spatial averages is discussed in the next section. 

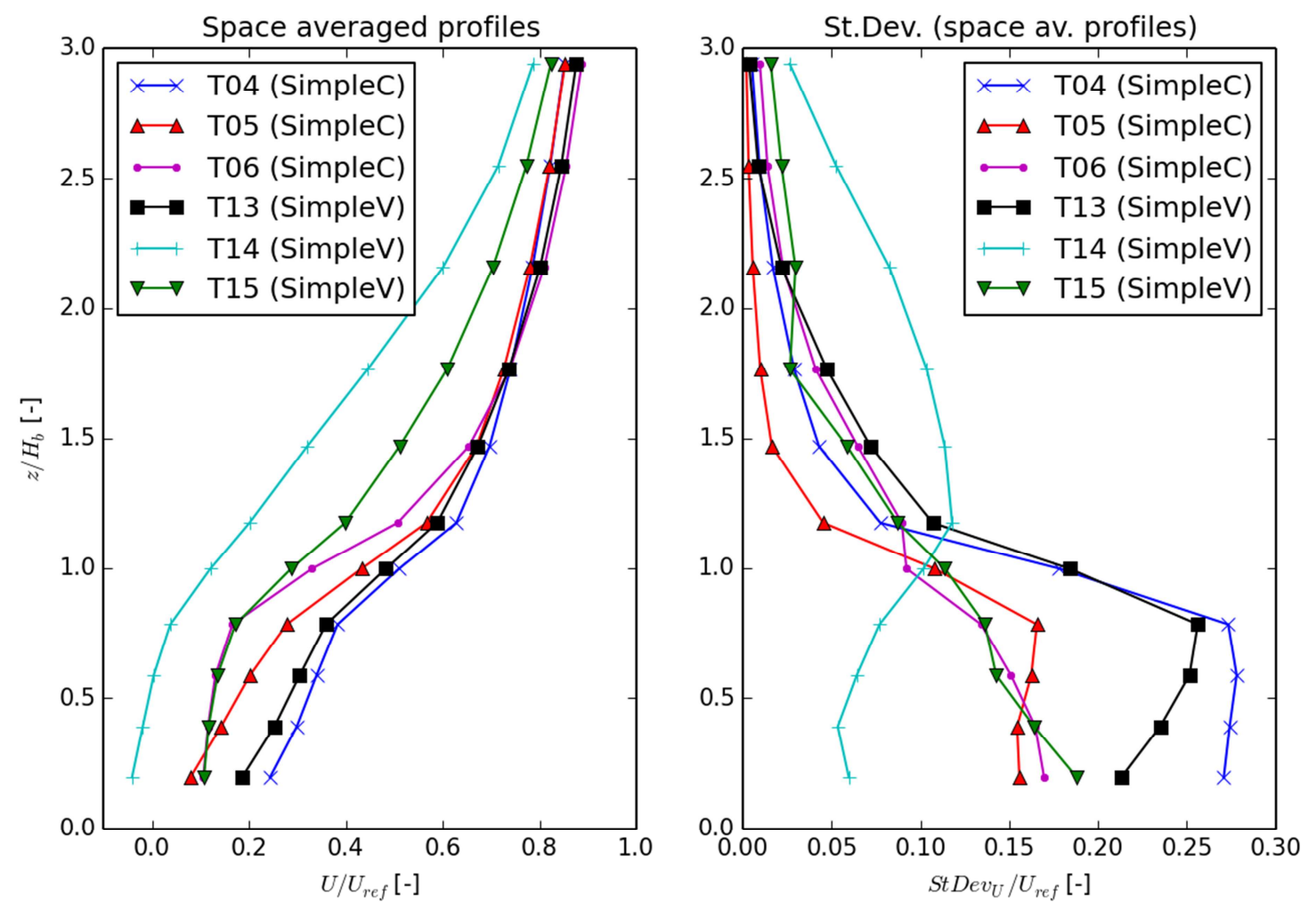

Figure 10 Spatially averaged vertical profiles of velocity (left), and standard deviation of the average profile (right); CFD simulations in the smaller high-resolution domain; the maximum building height in SimpleV is at $1.59 \mathrm{H}_{\mathrm{b}}$

\subsection{Representativeness of spatial averages}

The results presented in the previous sections highlighted the influence of local geometrical features on the spatially averaged profiles. As explained in section 2, the wind tunnel measurements were carried out mostly around the central intersection with a high spatial resolution grid. In order to assess the representativeness of spatial averages, a further series of measurements was carried out in a larger, but lower resolution, grid (labelled LDA-2 in Table 1). The analysis was then extended using the results from the CFD simulations.

Figure 11 presents a summary of the results from the second series of experiments. The 6 test cases are the same as in the first series, with experiments on both SimpleC $\left(\mathrm{T} 32,0^{\circ}\right.$; $\left.\mathrm{T} 33,90^{\circ} ; \mathrm{T} 34,45^{\circ}\right)$ and SimpleV $\left(\mathrm{T} 38,0^{\circ} ; \mathrm{T} 39,90^{\circ} ; \mathrm{T} 40,45^{\circ}\right)$. The six test cases are equivalent, respectively, to T04, T05, T06, T13, T14 and T15 in the first series.

Whilst the single profiles show very different results for the different test cases (see for example the velocity profiles taken at the centre of the model, Figure 11-left), the 
differences in the spatially averaged profiles are greatly reduced, compared with the first set of experiments (see figure 9 and 10).
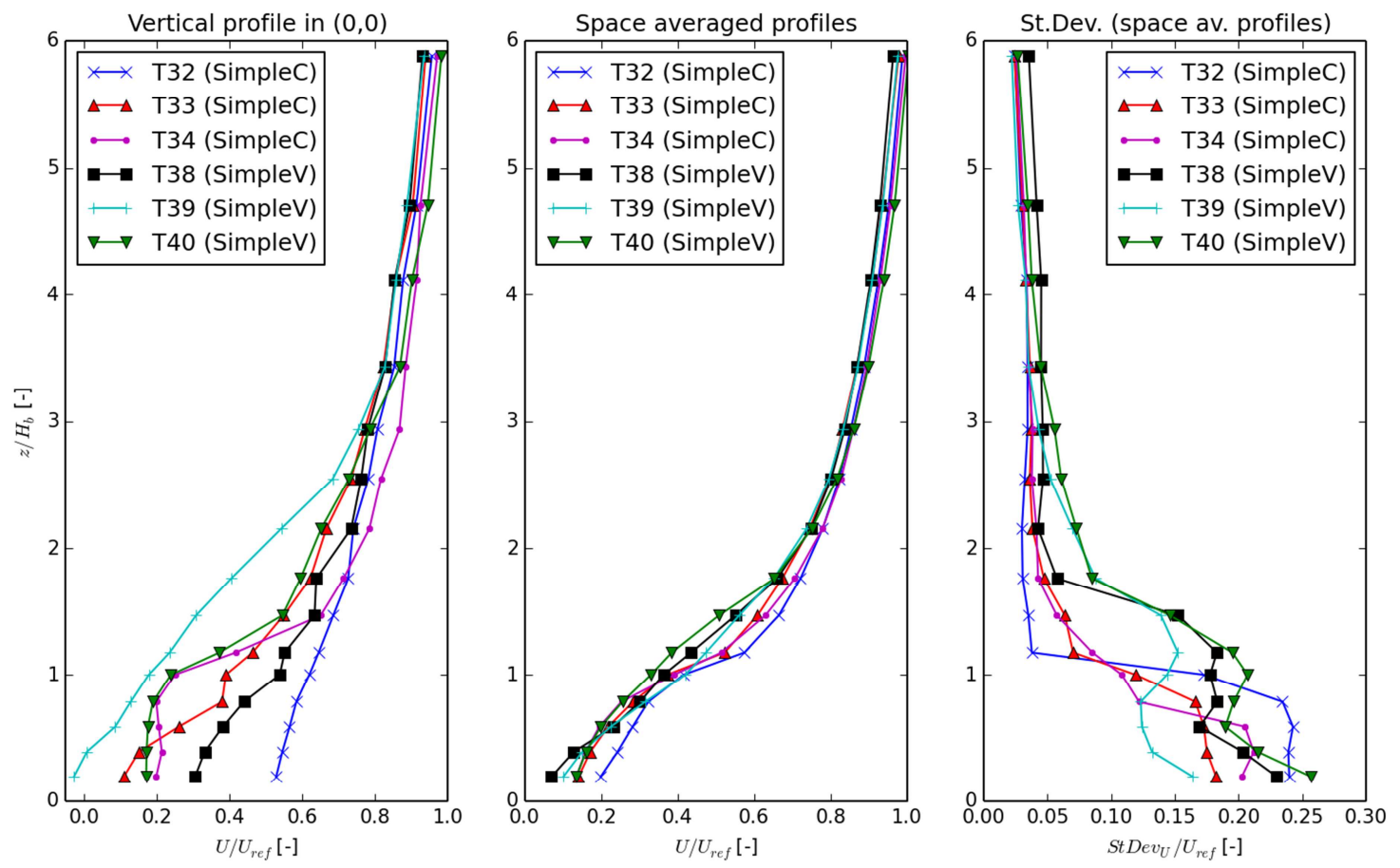

Figure 11 Vertical profile of velocity at $(x, y)=(0,0)$ (left); spatially averaged vertical profiles of velocity (centre), and standard deviation of the average profile (right); wind tunnel measurements, larger low-resolution domain; the maximum building height in SimpleV is at $1.59 H_{\mathrm{b}}$

The extended vertical reach of the profiles provided a better appreciation of behaviour above the canopy. The differences between spatially averaged velocities are generally negligible at $z=260 \mathrm{~mm}\left(2.55 \mathrm{H}_{\mathrm{b}}\right)$ and above in all cases. Even the variability of the single profiles (measured by the standard deviation in Fig. 11-right) for the SimpleC cases (where the buildings are all $102 \mathrm{~mm}$ high) is very low from $260 \mathrm{~mm}$ and upwards. As expected the variability for SimpleV is still significant, yet small, at $260 \mathrm{~mm}$ and remains so until around $z$ $=350 \mathrm{~mm}\left(3.43 H_{\mathrm{b}}\right.$, or $2.16 \mathrm{H}_{\max }-$ for reference, the tallest buildings in SimpleV are $162 \mathrm{~mm}$ high).

Spatially averaged mean velocity profiles from the wind tunnel (2 resolutions; LDA-1: T04, T05, T06, T13, T14 and T15; LDA-2: T32, T33, T34, T38, T39 and T40; see Table 1) and CFD (2 domains) are compared in Figure 12. The generally good agreement between the measurements and the simulations is clear, as highlighted in Section 3. The greatest differences appear in the $90^{\circ}$ cases, especially for the SimpleV model, where CFD and wind tunnel clearly to disagree more than in the other cases. These differences can be due, again, to the complex flow conditions imposed by the tall, upwind buildings on the lower part of 
the flow field. In fact, the tall buildings affect, in particular, the central area of the model (where the grid LDA-1 is located) and this is reflected in the disagreement between measurement and simulations for the T14 case (see Figure 12, bottom centre). On the larger domain (LDA-2 - case T39) the spatial averages are less affected and this results in smaller differences between CFD and wind tunnel.
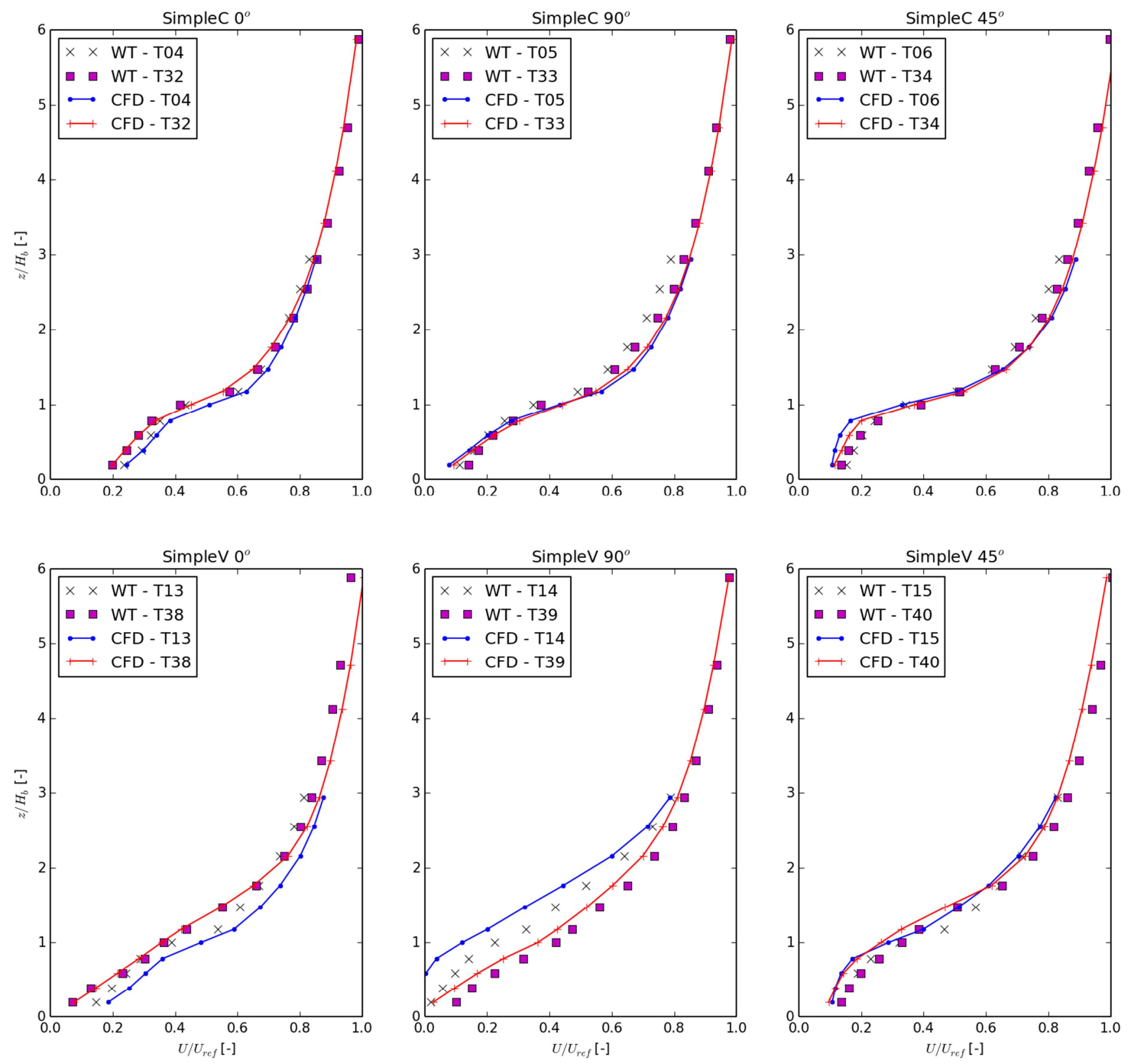

Figure 12 Spatially averaged vertical profiles of velocity from wind tunnel and CFD data; the maximum building height in SimpleV is at $1.59 \mathrm{H}_{\mathrm{b}}$

The use of one spatial domain or the other does not appear to make much difference to the spatially averaged profiles for the SimpleC model (the differences are mostly within $\pm 10 \%$ ), where all the buildings have the same height. Remarkably, the spatially averaged vertical profiles for SimpleV at $45^{\circ}$ do not change much, either, despite the fact that larger discrepancies were expected due to the larger blockage ratio. Larger differences can be seen, though, for SimpleV at $0^{\circ}$ and $90^{\circ}$ wind directions. 
The comparisons discussed above provided some confidence in the CFD results for the mean velocity fields. We therefore extended the analysis of the influence of the spatial domain to the full CFD data set, which, quite obviously, has a much higher resolution than the wind tunnel measurements. In particular, three progressively larger spatial domains were considered: these are labelled as 'CFD1', 'CFD2' and 'CFD3' in Figure 3. The boundaries of the CFD1 domain are roughly the same as the LDA-1 measurement grid, while the extension of CFD2 is comparable to the LDA-2 grid. CFD3 encompasses the whole building array, except the outermost building rows. The resulting spatially averaged profiles are shown in Figure 13.
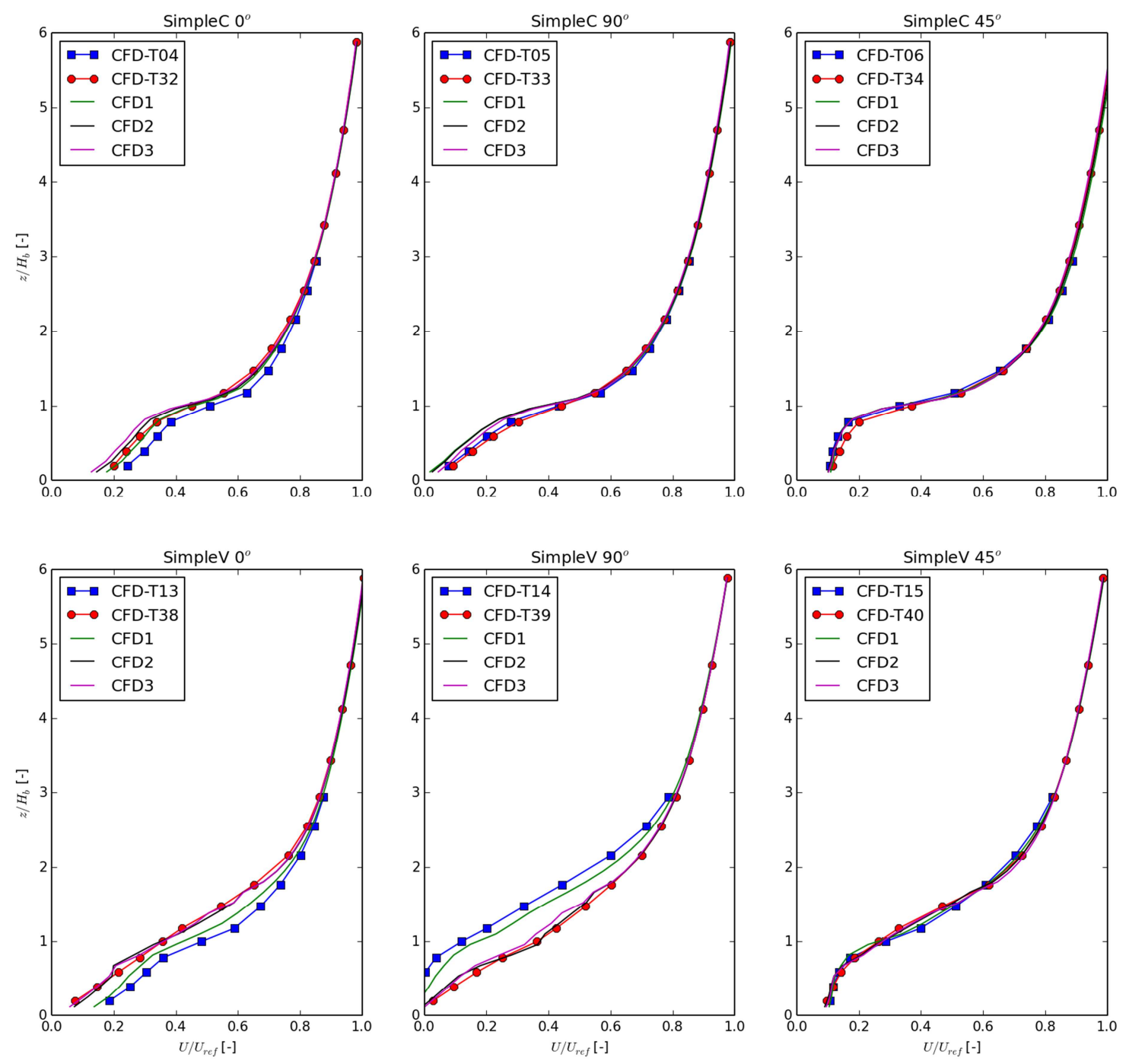

Figure 13 The effect of the spatial domain used to evaluate spatially averaged vertical profiles of mean velocity from the CFD results; the maximum building height in SimpleV is at $1.59 H_{\mathrm{b}}$ 
The results presented in Figure 13 confirm the analysis of the wind tunnel data from Figure 12. Indeed, without the inherent variability inherent in the experimental data, the remarkable similarities between the vertical profiles for SimpleC $90^{\circ}$, SimpleC $45^{\circ}$ and SimpleV $45^{\circ}$ can be appreciated all the more.

Results from spatial domains with similar boundaries (CFD1 vs. LDA-1 and CFD2 vs. LDA-2) are extremely close, confirmation that the differences in the spatial averages of the wind tunnel data are not caused by the lower spatial resolution of the measurement grids compared with the CFD data. The single element that causes the largest differences in the calculated profiles is the passage between the spatial domain that includes the four central buildings only and the wider spatial domains that contain a more diverse mix of building heights (see Figure 13-bottom left and centre). The usual suspect for such differences is, again, the influence of local features, tall buildings in particular, just upwind of the domain. Their effect is almost cancelled when the spatial boundaries are wider and include the tall buildings themselves.

\section{Discussion and conclusions}

In this paper we have presented an experimental and numerical analysis of the impact of some morphological parameters on the flow within and above an urban canopy. Experimental studies on simplified and idealised geometries constitute the bulk of the experimental data used today as a basis for model development and validation. While some past efforts attempted to study the influence of geometrical parameters other than the building height and building density (Cheng and Castro, 2002; Xie and Castro, 2006; Xie et al., 2008), their conclusions, while useful, cannot be easily generalised for real urban settings.

On the other hand, a number of recent studies have treated real urban areas. The results have been extremely useful in identifying shortcomings of current modelling approaches (Carpentieri et al., 2012) and important phenomena likely to have a major impact on flow and dispersion (Carpentieri et al., 2009; Carpentieri and Robins, 2010). However, more systematic approaches are needed to make possible the quantification and identification of the relative importance of the various parameters involved.

The present study is a first attempt in this direction. Many current modelling approaches would predict the same results in the six case studies presented here (two models by three wind directions), as all six cases have the same building density and mean building height. The frontal area densities however differ for the three wind direction, but are the same for the two models, SimpleC and SimpleV, so that only mathematical approaches that make use 
of this parameter would differentiate between the three wind directions, but even then not between the two models.

Results presented in Section 4 have demonstrated that the differences in the spatially averaged vertical profiles are actually significant in all six cases, whether studied experimentally or numerically. Besides the building height variability, other morphological features proved to be a significant factor in shaping flow and dispersion at the local to neighbourhood scale in the urban canopy and directly above: building aspect ratio (or, conversely, the street canyon aspect ratio), the angle between the street canyons and the oncoming wind, and local geometrical features such as, for example, the presence of much taller buildings immediately upwind of the area studied.

The first aspect (i.e., building/canyon aspect ratio and its angle with respect to wind direction), to our knowledge, is currently taken into account only by the SIRANE model (Soulhac et al., 2011) in the calculation of the average velocity within the street canyons (e.g. Soulhac et al., 2008). However, no modifications to the flow immediately above the canopy are incorporated in this model.

The latter aspect (i.e. the effect of tall buildings) has been sporadically studied in the past, and previous experiments have already highlighted its importance (Carpentieri et al., 2009; Carpentieri and Robins, 2010; Heist et al., 2009; Brixey et al., 2009). However, no modelling approaches have been proposed to take account of associated flow and dispersion phenomena. The results presented here, in particular in Section 4.3, showed that local geometrical features such as these can have a major impact on the flow within and above the urban canopy. This impact is likely to be localised in extent, probably with negligible influence on the neighbourhood scale flow. Nevertheless, since they can affect the area where sources are located and the mass exchanges in the local streets/intersections, they can have an important impact on neighbourhood scale dispersion of pollutants or other hazardous substances.

Further experimental and numerical studies will be needed to understand in more detail how to model spatially averaged wind profiles adequately in urban areas and, more generally, urban flows within the canopy and the roughness sub-layer. The present study has highlighted some of the parameters that need to be taken into account both in further studies and by incorporation into mathematical models, either in the extension of current or the development of new parameterisations. The difficulties and dangers inherent in calculating $u^{*}$ from velocity measurements (as is often done) have already been highlighted by Cheng and Castro (2002) and the current study shows these to be exacerbated in more realistic geometries. In the light of this, the use of a single scaling parameter for estimating spatially averaged profiles in the canopy and the roughness sub-layer does not seem very 
realistic. Of course, many current modelling approaches rely on so doing, so understanding the associated uncertainty is perhaps as important as deriving new methodologies.

The experimental and numerical database discussed here, while substantial, could be further developed and enhanced by future research. A better characterisation of turbulence, both in space and time (e.g. through 3-component measurements and particle image velocimetry), could help understand more deeply the flow within the canopy and its structure. Measuring the vertical components of velocity and turbulence, in particular, would allow a deeper characterisation of the shear layer at the top of the urban canopy, a better understanding of momentum transfer mechanisms (especially the vertical transfer between the canopy and flow above), an analysis of the TKE budget and, possibly, a more reliable estimation of the depth of the roughness sub-layer (RSL).

The latter (RSL depth) has been attempted using the available data set and, specifically, the longitudinal components of velocity and turbulence. We used the commonly accepted (although quite vague) definition of the top of the RSL as the point where the velocity and turbulence profiles collapse. A translation in quantitative terms of this definition is not trivial. In our case, we found that the inherent variability of the measurements was approximately of the same order of magnitude as the variability induced by the urban model in the flow well above the canopy. To give a numerical quantification, however, we decided to use an arbitrary definition of "collapsed profile": since the normal uncertainty of measurements in the wind tunnel is thought to be within the order of $\pm 10 \%$, we considered the top of the RSL the height where the velocity or turbulence values of the measured profiles are all within $\pm 10 \%$ of the average value. An alternative definition would be to consider the standard deviation of the measured values at a particular height to be above the RSL when this is lower than, say, $5 \%$. We must point out that using one definition or the other does give different (sometimes significantly) results, as does the arbitrary choice of the threshold limit (10\%? 11\%? $8 \%$ ?), for the reason we mentioned above, that the inherent uncertainty is of the same order of magnitude as the geometry-induced variability above the canopy. Also, different data-sets (e.g, high resolution wind tunnel measurements in the central part of the model, lower resolution measurements in the larger domain, or CFD data) give slightly different results. Using the above definition applied to the mean velocity profiles, we found that the RSL height varied between $z_{*}=2.16 H_{\mathrm{b}}$ to $z *=3.43 H_{\mathrm{b}}$. This is consistent with previous studies (see, e.g., Cheng et al., 2007). The lowest values are, of course, for the SimpleC model, where we also observed an influence of the wind direction (lowest value for $0^{\circ}$, highest value for $45^{\circ}$ ). For the SimpleV model, while observing consistently a deeper RSL, we did not see a significant dependence on the wind direction. This analysis is based on the mean velocity profiles measured in the larger, lower resolution, domain (where the vertical profiles extend to higher levels). The turbulence profiles never satisfy the arbitrary threshold, usually showing a variability on the order of $\pm 11-15 \%$ compared to the average values. 
Further concentration measurements will also be useful for characterising dispersion properties in these types of building arrays. The CFD modelling applied in the current study has proved to be very useful and accurate for complementing the wind tunnel measurements as far as the mean velocity profiles were concerned. However, for a fully reliable approach that would also be valid for the anisotropic turbulence fields, other more computationally demanding approaches (such as large eddy simulation) may be necessary.

\section{Acknowledgements}

We gratefully acknowledge the technical support by Dr Paul Hayden, at the University of Surrey, during the wind tunnel experiments, and useful discussions with Dr Zheng-Tong Xie (University of Southampton) for CFD modelling. This research has been funded by the European Commission under the FP7-People Programme (Marie-Curie Fellowship), grant PIEF-GA-2008-219318.

\section{References}

Arnold SJ, ApSimon H, Barlow J, Belcher S, Bell M, Boddy JW, Britter R, Cheng H, Clark R, Colvile RN, Dimitroulopoulou S, Dobre A, Greally B, Kaur S, Knights A, Lawton T, Makepeace A, Martin D, Neophytou M, Neville S, Nieuwenhuijsen M, Nickless G, Price C, Robins A, Shallcross D, Simmonds P, Smalley RJ, Tate J, Tomlin AS, Wang H, Walsh P (2004) Introduction to the DAPPLE air pollution project. Sci Total Environ 332:139-153

Belcher SE (2005) Mixing and transport in urban areas. Philos T Roy Soc A 363:2947-2968

Bentham T, Britter R (2003) Spatially averaged flow within obstacle arrays. Atmos Environ 37:20372043

Blocken B, Stathopoulos T, Carmeliet J (2007). CFD simulation of the atmospheric boundary layer: wall function problems. Atmos Environ 41:238-252

Brixey LA, Heist DK, Richmond-Bryant J, Bowker GE, Perry SG, Wiener RW (2009). The effect of a tall tower on flow and dispersion through a model urban neighbourhood. Part 2. Pollutant dispersion. J Environ Monit 11:2171-2179

Caretto L, Gosman A, Patankar S, Spalding D (1973) Two calculation procedures for steady, threedimensional flows with recirculation. Proceedings of the Third International Conference on Numerical Methods in Fluid Mechanics, volume 19 of Lecture Notes in Physics, 60-68

Carpentieri M, Hayden P, Robins AG (2012a) Wind tunnel measurements of pollutant turbulent fluxes in urban intersections. Atmos Environ 46:669-674

Carpentieri M, Robins AG (2010) Tracer flux balance at an urban canyon intersection. BoundaryLayer Meteorol 135:229-242

Carpentieri M, Robins AG, Baldi S (2009) Three-dimensional mapping of air flow at an urban canyon intersection. Boundary-Layer Meteorol 133:277-296

Carpentieri M, Salizzoni P, Robins A, Soulhac L (2012b). Evaluation of a neighbourhood scale, street network dispersion model through comparison with wind tunnel data. Environ Modell Softw 37:110-124

Chang JC, Hanna SR (2004) Air quality model performance evaluation. Meteorol Atmos Phys 87:167196 
Cheng H, Castro IP (2002) Near wall flow over urban-like roughness. Boundary-Layer Meteorol 104:229-259

Cheng $\mathrm{H}$, Hayden P, Robins AG, Castro IP (2007). Flow over cube arrays of different packing densities. $J$ Wind Eng Ind Aerod 95:715-740

Coceal O, Belcher S (2004) A canopy model of mean winds through urban areas. Q J R Meteorol Soc 130:1349-1372

Davidson MJ, Mylne KR, Jones CD, Phillips JC, Perkins RJ, Fung JCH, Hunt JCR (1995) Plume dispersion through large groups of obstacles - a field investigation. Atmos Environ 29:3245-3256

Finnigan J (2000) Turbulence in plant canopies. Annu Rev Fluid Mech 32:519-572

Franke J, Hellsten A, Schlünzen H, Carissimo B (Eds.) (2007) Best practice guideline for the CFD simulation of flows in the urban environment. COST Action $732.52 \mathrm{pp}$.

Garbero V, Salizzoni P, Soulhac L (2010). Experimental study of pollutant dispersion within a network of streets. Boundary-Layer Meteorol 136:457-487

Grimmond CSB, Oke TR (1999) Aerodynamic properties of urban areas derived from analysis of surface form. J Appl Meteorol 38:1262-1292

Hamlyn D, Britter R (2005) A numerical study of the flow field and exchange processes within a canopy of urban-type roughness. Atmos Environ 39:3243-3255

Hangan H (1999). Wind driven rain studies. A C-FD-E approach. J Wind Eng Ind Aerod 81:323-331.

Harms F, Leitl B, Schatzmann M, Patnaik G (2011). Validating LES-based flow and dispersion models. J Wind Eng Ind Aerod 99:289-295

Heist DK, Brixey LA, Richmond-Bryant J, Bowker GE, Perry SG, Wiener RW (2009). The effect of a tall tower on flow and dispersion through a model urban neighbourhood. Part 1. Flow characteristics. J Environ Monit 11:2163-2170

Jerram N, Perkins RJ, Fung JCH, Davidson MJ, Belcher SE, Hunt JCR (1995) Atmospheric flow through groups of buildings and dispersion from localised sources, in: Cermak J, Davenport A, Plate E, Domingos X (Eds.), Wind climate in cities. Kluwer, 109-130.

Kastner-Klein P, Plate EJ (1999). Wind-tunnel study of concentration fields in street canyons. Atmos Environ 33:3973-3979

Klein P, Leitl B, Schatzmann M (2011). Concentration fluctuations in a downtown urban area. Part II: analysis of Joint Urban 2003 wind-tunnel measurements. Environ Fluid Mech 11:43-60

Launder BE, Spalding DB (1974) The numerical computation of turbulent flows. Comp Method Appl Mech Eng 3:269-289

MacDonald R, Carter S, Slawson P (2000) Measurements of mean velocity and turbulence statistics in simple obstacle arrays at 1:200 scale. Thermal Fluids Report 2000-01, University of Waterloo, $130 \mathrm{pp}$.

MacDonald R, Griffiths R, Hall D (1998a) A comparison of results from scaled field and wind tunnel modelling of dispersion in arrays of obstacles. Atmos Environ 32:3845-3862

MacDonald R, Griffiths R, Hall D (1998b). An improved method for estimation of surface roughness of obstacle arrays. Atmos Environ 32:1857-1864

Milliez M, Carissimo B (2007). Numerical simulations of pollutant dispersion in an idealized urban area, for different meteorological conditions. Boundary-Layer Meteorol 122:321-342

OpenFOAM (2009) OpenFOAM - The Open Source CFD Toolbox. User Guide, Version 1.6. OpenCFD. 203 pp.

Ratti C, Di Sabatino S, Britter R (2006) Urban texture analysis with image processing techniques: winds and dispersion. Theor Appl Climatol 84:77-90

Ratti C, Di Sabatino S, Britter R, Brown M, Caton F, Burian S (2002) Analysis of 3-D urban databases with respect to pollution dispersion for a number of European and American cities. Water Air Soil Poll Focus 2:459-469

Robins A (2003). Wind tunnel dispersion modelling some recent and not so recent achievements. J Wind Eng Ind Aerod 91:1777-1790 
Salizzoni P, Soulhac L, Méjean P (2009). Street canyon ventilation and atmospheric turbulence. Atmos Environ 43:5056-5067

Soulhac L, Perkins RJ, Salizzoni P (2008). Flow in a street canyon for any external wind direction. Boundary-Layer Meteorol 126:365-388

Soulhac L, Salizzoni P, Perkins RJ (2011). The model SIRANE for atmospheric urban pollutant dispersion; part I, presentation of the model. Atmos Environ 45:7379-7395

Theurer W (1999) Typical building arrangements for urban air pollution modelling. Atmos Environ 33:4057-4066

Tominaga Y, Mochida A, Yoshie R, Kataoka H, Nozu T, Yoshikawa M, Shirasawa T (2008) AlJ guidelines for practical applications of CFD to pedestrian wind environment around buildings. J Wind Eng Ind Aerod 96:1749-1761

Tominaga Y, Stathopoulos T (2013). CFD simulation of near-field pollutant dispersion in the urban environment: A review of current modeling techniques. Atmos Environ 79:716-730

Xie Z, Castro IP (2006) LES and RANS for turbulent flow over arrays of wall-mounted obstacles. Flow Turbul Combus 76:291-312.

Xie Z, Coceal O, Castro IP (2008). Large-eddy simulation of flows over random urban-like obstacles. Boundary-Layer Meteorol 129:1-23.

Yee E, Biltoft C (2004) Concentration fluctuation measurements in a plume dispersing through a regular array of obstacles. Boundary-Layer Meteorol 111:363-415 